\title{
Journal of Cardiovascular Magnetic

\section{Quantification of 4D left ventricular blood flow organization in normal and failing hearts}

\author{
Carl Johan Carlhäll*1, Jonatan Eriksson 1,2, Petter Dyverfeldt ${ }^{1,2}$, \\ Tino Ebbers 1,2 , Jan Engvall ${ }^{1,2}$ and Ann F Bolger ${ }^{3}$
}

\author{
Address: ${ }^{1}$ Linköping University, Linköping, Sweden, ${ }^{2}$ Center for Medical Image Science and Visualization (CMIV), Linköping, Sweden and \\ ${ }^{3}$ University of California San Francisco, San Francisco, CA, USA \\ * Corresponding author
}

from I3th Annual SCMR Scientific Sessions

Phoenix, AZ, USA. 21-24 January 2010

Published: 21 January 2010

Journal of Cardiovascular Magnetic Resonance 2010, I 2(SuppI I):P70 doi:I0.1 I86/I532-429X-I2-SI-P70

This abstract is available from: http://jcmr-online.com/content/I2/SI/P70

(c) 2010 Carlhäll et al; licensee BioMed Central Ltd.

\section{Objective}

To measure the volume and distribution of separate flow components in normal and dysfunctional left ventricles (LV).

\section{Background}

The forces created by blood flow dictate a continuous remodeling of cardiac structures. In the normal heart this mechanism interactively creates an optimal geometry for efficient flow [1]. In various cardiac disorders such as LV dysfunction, however, the normal flow organization may be altered, leading to negative remodelling. The description and quantification of the true 4D (3D+time) blood flow organization in dysfunctional LVs remains incomplete.

\section{Method}

Seven dilated cardiomyopathy (DCM) patients (4 female, aged $52 \pm 14$ years [mean \pm SD] $)$ and six healthy subjects ( 3 female, aged $58 \pm 4$ years) were studied. Three-directional, 3-dimensional cine phase-contrast-MRI velocity data and morphological b-SSFP long- and short-axis images were acquired on a clinical 1.5 T MRI scanner (Philips Achieva). The LV endocardium was segmented (http://segment.heiberg.se, [2]) from the short-axis images at the times of isovolumetric contraction (IVC) and isovolumetric relaxation (IVR). Pathlines were emitted from the IVC LV blood volume and traced forward and backward in time until IVR, thus covering the entire car- diac cycle. The IVR volume was used to determine if and where the traces left the LV. This information was used to automatically separate the pathlines into four different LV flow components $[3,4]$ (Table 1, Figure 1).
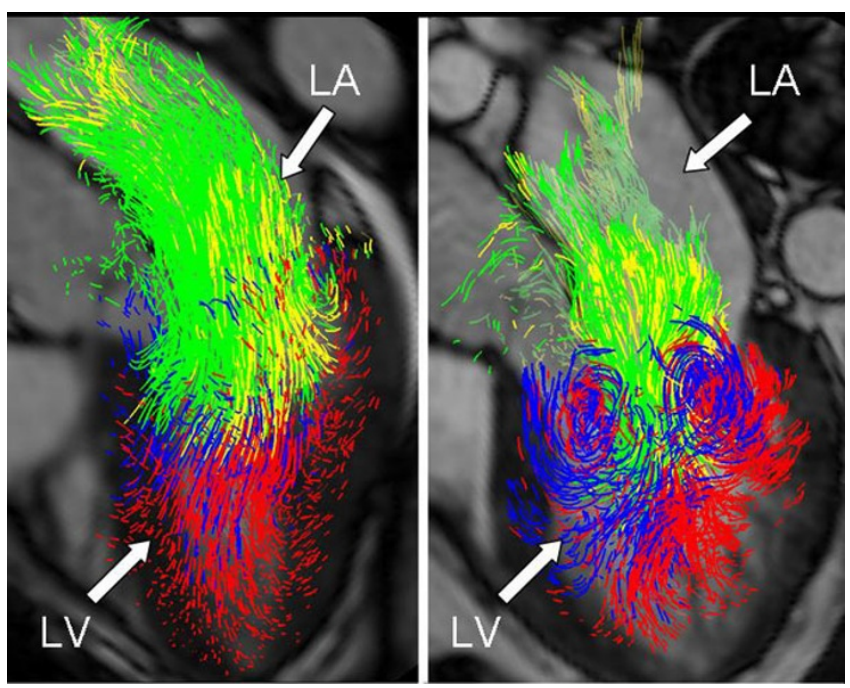

Figure I

Pathline visualization of LV blood flow organization at early diastole in a healthy $\mathbf{5 8}$ year old female (left panel) and a $6 \mathrm{I}$ year old female with dilated cardiomyopathy (right panel). Green, direct flow; yellow, retained inflow; blue, delayed ejection flow; and red, residual volume. LA, left atrium; and LV, left vemtricle. 
Table I: Components of LV blood flow

\begin{tabular}{ll}
\hline Component & Description \\
\hline Direct Flow & Blood that enters and leaves the LV within the same cardiac cycle \\
Retained Inflow & Blood that enters but does not leave the LV within the same cardiac cycle \\
Delayed Ejection Flow & Blood that leaves but does not enter the LV within the same cardiac cycle \\
Residual Volume & Blood that resides in the LV more than two cardiac cycles \\
\hline
\end{tabular}
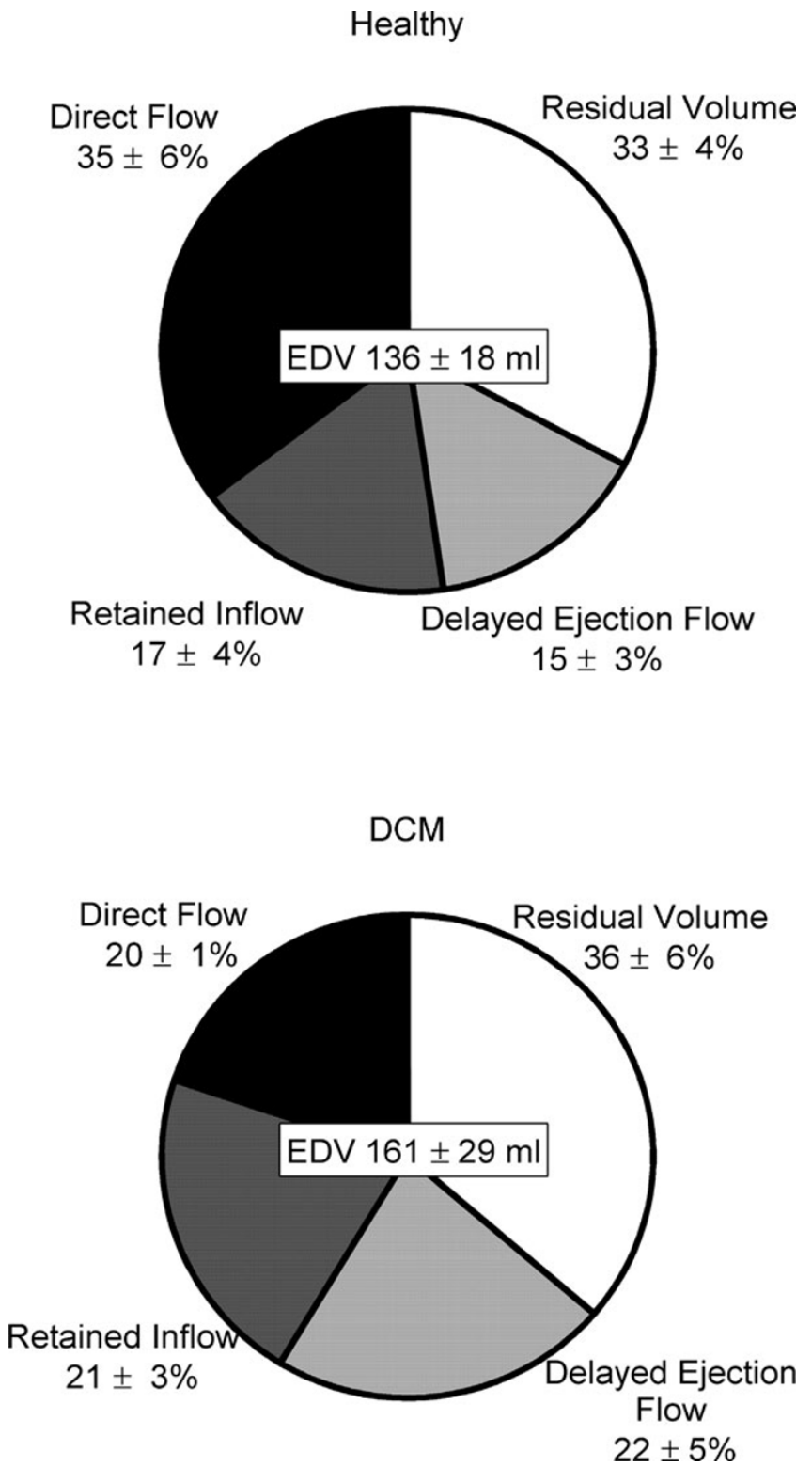

Figure 2

Illustration of the four LV flow components as a percentage of LV end-diastolic volume (mean \pm SD) in the six healthy subjects and seven patients with dilated cardiomyopathy.

\section{Results}

There was no difference in heart rate between DCM and healthy subjects ( $58 \pm 11$ vs $64 \pm 8 \mathrm{bpm}, \mathrm{NS})$. The LV ejection fraction was smaller and LV end-diastolic diameter was larger in DCM compared to healthy subjects $(43 \pm 5$ vs $59 \pm 2 \%, \mathrm{p}<0.001$, and $60 \pm 5$ vs $45 \pm 3 \mathrm{~mm}, \mathrm{p}<0.001$, respectively). The direct flow/end-diastolic volume (EDV) ratio was lower and the delayed ejection flow/EDV ratio was higher in the DCM compared to healthy subjects (20 \pm 1 vs $35 \pm 6 \%, \mathrm{p}<0.001$, and $22 \pm 5$ vs $15 \pm 3 \%, \mathrm{p}<0.01$, respectively) (Figure 2 ).

\section{Conclusion}

Multidimensional flow analysis can measure the compartmentalization of blood transiting the beating LV. The present findings imply that the highly organized flow in and out of normal LVs is demonstrably altered in DCM, even with only moderate LV dilation and dysfunction. Such measures may contribute not only to our understanding of the pathophysiology and diagnosis of disease, but also to the optimization of therapy.

\section{References}

I. Richter Y, Edelman E: Circulation 2006, I | 3:2679-2682.

2. Heiberg E, et al.: Comput Cardiol 2005, 32:599-602.

3. Eriksson J, et al: ISMRM flow workshop 2009:47.

4. Bolger AF, et al.: JCMR 2007, 9:74I-747. 